\title{
1910 : La Seine en crue paralyse la capitale
}

\author{
par M. Ambroise-Rendu \\ Journaliste, Conseiller en environnement urbain
}

\section{I — UNE VILLE DU XXÈME SIÈCLE}

En 1910 les Français, et au premier rang d'entre eux les Parisiens, sont fiers des progrès techniques qui changent le paysage et le confort de la capitale. Paris bénéficie en effet d'une modernisation accélérée comme il ne s'en était pas produit depuis le préfet Haussmann. Il ne s'agit pas d'urbanisme - l'essentiel est fait - mais d'aménités urbaines qui montrent aux citadins qu'ils sont bien entrés dans le XXème siècle.

Les galeries d'égouts qui 50 ans plus tôt ne mesuraient que 140 kilomètres courent à présent sous 1200 kilomètres de rues et de boulevards. Plus de la moitié des immeubles parisiens (45 000 sur 80000$)$ y sont raccordés et leurs eaux usées filent vers les champs d'épandage et la station d'épuration d'Achères. Dans ces galeries visitables on a suspendu les conduites d'eau potable, celles de l'eau industrielle, les tuyaux où circulent les messages pneumatiques et les câbles téléphoniques.

Les propriétaires d'immeubles non encore raccordés font appel à des équipes spéciales qui, la nuit, le plus discrètement possible, vidangent les fosses d'aisance à l'aide de pompes à vapeur.

Les déchets domestiques sont rassemblés dans des récipients normalisés et rendus obligatoires par le préfet Poubelle, puis ramassés quotidiennement par 520 tombereaux attelés. Ceux-ci se dirigent alors vers quatre usines situées en proche banlieue où les détritus sont soit incinérés soit transformés en engrais.
Pour l'énergie, Paris s'enorgueillit d'offrir à ses habitants trois sources modernes et différentes. Produit par 9 usines brûlant du charbon, le gaz est distribué en ville par la Société du Gaz de Paris. Il alimente 53000 lampadaires municipaux et 630000 foyers privés. L'air comprimé "fabriqué" dans le XIIIème arrondissement (il en reste un intéressant bâtiment encore visible quai Panhard et Levassor) fait tourner 5800 pendules plantées sur les trottoirs. A Paris on peut se passer d'une montre puisque l'heure est affichée partout. Des canalisations vont également porter l'air sous pression à 4000 ascenseurs d'immeubles ainsi qu'à de multiples machines fonctionnant dans les usines et les ateliers d'artisans. Troisième possibilité, l'électricité qui est fournie par trois sociétés concessionnaires : Continental-Edison, Victor Popp et la Société d'Eclairage et de Force. Chacune possède ses usines et son secteur de distribution. Au total 14 usines, une quinzaine de sous-stations, des milliers de transformateurs et 1300 kilomètres de câbles alimentent déjà 67000 abonnés dont le premier est évidemment l'Elysée. Mais certaines églises commencent à se raccorder et les curés bénissent - au propre comme au figuré- ces installations "féériques".

\section{II 日 L'ÂGE D'OR DU RAIL}

Dans le domaine des transports en commun la capitale est, dès cette époque, l'une des cités les mieux équipées de la planète. Appartenant à 11 compagnies différentes, de nombreux tramways de divers modèles desservent la plupart des

In 1910 Paris was in the middle of its modernization. Fresh water, drainage, gas, electricity, compressed air, rubbish disposal, telegraph, telephone, trams, underground and railways, all these amenities were already at the disposal of Parisians. To preserve the urban landscape most of the networks were undergnound and the factories outside of the city. All this protected from rain water. But the rise of the water level of the Seine and its ground water broke through its defenses. In a few days the water had flooded the factories on the embankments, rushed into the underground sites, came back up the sewers and overun the cellars. All the networks including those which supply energy were affected, paralysing the city even in areas far from the river. Besides the suffering inflicted on some Parisians and the inconvenience of the cleaning up operations imposed on a lot of people, the flooding of the Seine cost community a lot. But it accelerated the modernization of the capital and helped develop the idea of better prevention of floods. 
quartiers. Ils utilisent tous les modes de traction alors disponibles. La traction hippomobile, la vapeur, l'électricité, l'air comprimé. Sous la chaussée leur concurrent - qui finira par les éliminer - progresse comme une taupe : le métro. La première ligne du chemin de fer métropolitain à écartement normal mais à petit gabarit - la ligne Vincennes-Etoile - a été inaugurée dix ans auparavant à l'occasion de l'Exposition universelle. Depuis, cinq autres lignes ont été creusées et les Parisiens peuvent circuler sur 63 kilomètres. Quatre autres lignes sont en construction et leurs chantiers perforent la chaussée en maints endroits. Bientôt les voyageurs pourront parcourir sous terre ou en viaduc 83 kilomètres. En janvier 1910 les officiels ont justement inauguré la ligne 4, la première à oser passer sous la Seine entre la place de l'Odéon et la place du Châtelet.

Dans le secteur des chemins de fer, Paris reste au centre d'une véritable toile d'araignée. La ligne d'Orléans et du Midi arrive jusqu'à Orsay en passant par Austerlitz puis en se glissant sous la chaussée, le long de la Seine. A l'opposé le réseau de l'Ouest court également en bordure du fleuve et parvient jusqu'aux Invalides. Les voyageurs peuvent aussi débarquer à la Bastille, aux terminaux de Lyon, du Nord, de l'Est, de Saint-Lazare, de Montparnasse et de Denfert-Rochereau. Dix embarcadères pour les hommes tandis que 3 autres sont affectés aux denrées, matériaux et marchandises. Le rail vit son âge d'or.

Sur la Seine une compagnie de navigation exploite 39 kilomètres de lignes avec une flotille de plus de 100 bateaux et 47 pontons-stations sur lesquels passent 8 millions de passagers par an. Pendant le même temps des chalands tractés par des remorqueurs à cheminée rabattable déposent sur les quais 9 millions de tonnes de matériaux.

\section{III $\square$ LES COQUETTERIES D'UNE CAPITALE}

Paris est encore le premier carrefour de communications de l'Hexagone. A l'intérieur de la ville les correspondances urgentes circulent dans des conduites pneumatiques et sont livrées à domicile par les fameux "petits télégraphistes". Vers la province et l'étranger les messages pressés sont transmis par fil à partir de deux centraux. Ils expédient en moyenne 90000 télégrammes par jour soit 33 millions par an. Mais voici déjà le téléphone qui dispose de plusieurs centraux, dont un sous la Tour Eiffel, et qui fait le bonheur de 42000 privilégiés.

En ce début de siècle la capitale n'oublie pas qu'elle doit tenir son rang parmi les plus belles villes du monde. Tous les réseaux, y compris électriques et téléphoniques, sont donc soigneusement dissimulés aux regards. Autrement dit ils sont soit enterrés soit suspendus à la voute des égouts. Quant aux usines de production d'énergie et de traitement des ordures ménagères elles sont repoussées dans les bas quartiers des arrondissements riverains de la Seine comme le XIIème et le XIIIème ou, mieux encore, logées en banlieue, mais souvent à proximité du fleuve puisque c'est par là qu'arrive le charbon alimentant les fours à gaz et les générateurs électriques.

Bien entendu réseaux, centraux et usines sont à l'abri de la pluie et de la neige. L'étanchéité garantit que la machinerie urbaine fonctionnera par tous les temps. On a même prévu un éventuel débordement du fleuve. Tout a été calculé pour résister à une crue type 1876 dont on n'imagine pas que les niveaux puissent être dépassés. Au reste il existe un "Service hydrométrique" dont les prévisionnistes doivent, à partir des observations faites en amont, annoncer à l'avance la montée de la Seine dans la capitale. En cas de crue exceptionnelle on aura le temps d'élever les défenses nécessaires. Bref, Paris est une ville coquette, moderne et techniquement fiable.

Cependant elle reste fragile car de nombreux orifices dus aux chantiers des lignes de métro percent les chaussées. A la suite d'un incident au cours duquel, en septembre 1909, les débordements d'un égout ont inondé une galerie de métro en cours de forage, le président du Conseil lui-même, Aristide Briand, a pressé les ingénieurs de refermer ces "plaies" au plus vite.

\section{IV — LE FLEUVE À L'ASSAUT DE PARIS}

Pourtant Paris va se laisser surprendre. Entre le 20 et le 28 janvier 1910 la Seine monte littéralement à l'assaut de la capitale. Ses eaux dont le niveau s'élève sans cesse et bien au-delà de celui de 1876, finissent par submerger la plupart des défenses qui leur sont hâtivement opposées. Les prévisionnistes sont pris en défaut. Les eaux envahissent d'abord les quais bas, puis certains quais hauts (sur 7 kilomètres) et, de là, les quartiers contigus. La submersion des chaussées n'est pas dramatique en soi. On y pallie en lancant des embarcations pilotées par des matelots. Dans les rues les plus résidentielles comme celles du 7ème arrondissement, les militaires du Génie dressent des tréteaux qui, sur des centaines de mètres, font office de trottoirs surélevés. Dans Paris Venise on circule encore.

En revanche, l'inondation des quais empêche la Seine de remplir son rôle de voie d'eau. Passagers et marchandises notamment le charbon alimentant les usines productrices d'énergie - ne débarquent plus. Les établissements euxmêmes, souvent proches du fleuve, sont assiégés et leurs machines sont les premières noyées.

L'eau montant encore trouve ici et là des pertuis accidentels, mal défendus ou qu'on a oublié d'aveugler. Ainsi la Seine s'engouffre dans un puits d'aération du chantier de la ligne de métro dite Nord-Sud, boulevard Saint-Germain. En quelques heures, bousculant tous les barrages que les ouvriers tentent de lui opposer au fond, elle s'insinue jusqu'au-dessous de la cour de Rome, devant la gare SaintLazare. Là, profitant d'un autre puits, elle jaillit et forme un lac qui plonge les ingénieurs, les autorités et les Parisiens dans la stupeur. Personne n'attendait le fleuve à 1400 mètres de son lit.

La Seine va également envahir les galeries ferroviaires qui la longent sur la rive gauche, transformer la gare d'Orsay en piscine et, de là, se déverser dans les rues du 7ème arrondissement situées en contre-bas. Même accident à l'Hôtel de Ville dont les soubassements ont été, lors de la reconstruction, doublement défendus par un fossé et une ceinture de béton. Une aération oubliée suffit. Les caves de la mairie sont bientôt sous l'eau et toute la machinerie municipale, chauffage compris, s'arrête.

Enfin les égouts sont une providence pour la Seine vagabonde. Normalement l'eau qui se répand sur la chaussée (lorsqu'il pleut, quand on lave le pavé ou à l'occasion d'un incendie) est évacuée par les galeries souterraines et les collecteurs. En cas d'orage violent ou de crue l'eau est rejetée à la Seine par des pompes spéciales. Mais si la submersion est considérable et dure plusieurs jours ce dispositif ne suffit 
plus. Les égouts sont pleins à ras bord et ils font l'office inverse. C'est par eux que la Seine se répand d'abord sous la ville puis dans la ville. L'eau commence par s'infiltrer dans les caves et les sous-sols en se glissant le long des passages de câbles mal étanchés. Puis, le niveau montant toujours, l'eau dégorge des regards et inonde la chaussée. Tous les réseaux se trouvant au dessous du pavé sont alors menacés. Soit par l'humidité soit par les mouvements du sol.

La submersion prolongée d'une cité moderne engendre sa paralysie. C'est ce qui arrive à la capitale en 1910 .

\section{V — PREMIÈRE TOUCHÉE : LA NAVIGATION}

Le jeudi 20 janvier le niveau de la Seine est tel que les navettes de la Compagnie des bateaux parisiens ne passent plus sous les ponts. Elles interrompent leur service. Le vapeur qui effectue la liaison Paris-Londres n'appareille pas non plus. Le tirant d'air sous le pont de l'Alma n'est plus suffisant. Le lendemain ce sont les remorqueurs et les péniches qui viennent à quai : 250 bateaux sont immobilisés dans la traversée de l'agglomération parisienne. Plusieurs centaines de mariniers et de matelots sont en chômage et sans indemnité. Ils le resteront durant 45 jours. Voilà Paris privé de l'un de ses transports en commun et le premier port fluvial de France coupé de ses approvisionnements.

\section{VI — LA VILLE LUMIÈRE S'ENTÉNÈBRE}

Le vendredi 21 janvier à $22 \mathrm{~h} 53$ exactement toutes les horloges de Paris cessent de tourner. L'air comprimé n'arrive plus. Les compresseurs qui le produisent et l'expédient dans tout Paris par un réseau de 350 kilomètres de canalisations sont noyés quai de la Gare, dans le XIIIème arrondissement. Des milliers d'immeubles sont privés d'ascenseurs et le lendemain à l'aube une foule d'artisans s'apercoivent qu'ils ne peuvent plus utiliser leurs machines. L'usine ne reprendra du service qu'après 13 jours d'interruption totale. Mais, par suite de la rupture de certaines canalisations, quelques quartiers resteront sevrés d'air sous pression durant deux mois.

Ce jour-là aussi l'électricité commence à défaillir. Les lampadaires du pont Alexandre III et ceux de l'avenue Gambetta, tout là-haut dans le 20ème arrondissement, s'éteignent. Explication : comme pour l'air comprimé ce sont les usines fourrnissant le courant qui sont en cause. Deux d'entre elles situées à Vitry, en banlieue sud, baignent dans 60 centimètres d'eau.

Ce n'est que le début de graves difficultés. Sur les 14 usines alimentant Paris, 6 devront être stoppées et 3 autres auront de sérieuses difficultés. Certaines sous-stations se noient, les transformateurs grillent et l'humidité s'insinue dans les câbles. Même dans les quartiers qui restent au sec, l'éclairage public et nombre d'abonnés vont être fréquemment privés de courant. C'est le cas sur une grande partie de la rive gauche mais aussi dans les secteurs Palais-Royal, Bourse, Opéra, Châteaudun, Monceau, Saint-Lazare, Malesherbes, Clichy, Batignolles et Champs-Elysées. Certains devront attendre 4 mois avant d'être à nouveau alimentés convenablement.

La Société du gaz de Paris commence à flancher le lundi 24 janvier. Jusque-là elle s'est flattée de faire tourner ses 9 usines de production : 4 à l'intérieur de la ville, 5 en périphérie. Mais ce jour-là, l'usine d'Alfort est touchée par les eaux ; une seconde, située à Passy, va fermer et trois autres seront fortement handicapées. Toutes pâtissent de l'arrêt de la navigation puisque la majorité du combustible arrive par péniches.

Ensuite ce sont les 1800 kilomètres de canalisations qui, par endroit, vont subir des avaries. Les eaux provoquent des mouvements de sol qui, eux-mêmes, engendrent des ruptures de conduites. 5837 becs de gaz s'éteignent progressivement. Les employés de la S.G.P. y accrochent en secours des lampes à pétrole, à acétylène ou à alcool. Néanmoins chaque après-midi, dès 17 heures, une partie de la capitale (environ $10 \%$ ) plonge dans la pénombre, seulement éclairée par des lumignons de fortune.

Pour servir ses abonnés malgré tout, la Compagnie installe dans les rues des tuyaux provisoires : 1600 mètres au total. Malgré ces efforts 30000 abonnés sur 630000 (soit 4,76\%) seront momentanément privés de gaz.

\section{VII $\square$ ON REVIENT À LA TRACTION ANIMALE}

Sur les lignes de tramways ce sont les rames à traction électrique qui succombent les premières. Le 21 janvier le Bastille-Montparnasse est immobilisé, comme les lignes de banlieue desservant Vitry, Bonneuil, Champigny et SaintMaur. Les deux usines alimentant ces compagnies sont celles de Vitry et, on l'a vu, elles sont noyées. Le lendemain d'autres compagnies sont frappées et les voyageurs se heurtent à cette affichette : "Interruption du service pour cause d'inondation". Après les rames mues à l'électricité, ce sont les tramways actionnés par l'air comprimé qui rendent l'âme lorsque leur usine d'alimentation est touchée.

Restent les machines à vapeur. Celles qui roulent sur l'itinéraire Louvre-Versailles longent le quai de Passy inondé en soulevant des gerbes d'eau. Elles émerveillent les Parisiens qui les comparent à des torpilleurs. La Compagnie générale des omnibus, la plus importante de ces sociétés, ressort de ses dépôts un certain nombre de trams à vapeur pour remplacer les machines électriques et à air comprimé. Elle installe leur dépôt en plein vent, Place de la Bastille, mais en vain. Bientôt les motrices manquent de charbon, d'eau pure et de graisse.

On en revient aux omnibus hippomobiles car les chevaux continuent à tirer leur charge même avec de l'eau jusqu'à mijambe. Partout les transports urbains reculent. On craint aussi les effondrements de chaussées fragilisées par la montée des eaux. Certaines lignes ne reprendront leur trafic qu'au bout de 10 semaines.

\section{VIII — LE MÉTRO NE SUPPORTE PAS L'HUMIDITÉ}

Le dimanche soir 23 janvier, vers minuit, les Parisiens sortant des spectacles trouvent les stations de métro Châtelet, Saint-Michel et Odéon plongées dans le noir. Un court-circuit a fait sauter les plombs : l'eau s'infiltre le long du ballast de la ligne 4 , celle qui passe sous la Seine. Une deuxième ligne, la 6 (Italie-Nation), est également fermée. L'usine qui l'alimente, Quai de la Rapée, est noyée. Le métro que ses ingénieurs affirmaient à l'abri de tout, flanche donc lui aussi. Après la 6 et la 4 c'est la 1 puis la 2 qui sont touchées malgré les pompes qui, jour et nuit, combattent les infiltrations. 5 lignes sur 6 seront finalement interrompues : la 1 à la Concorde, la 3 à l'Opéra, la 4 sous la Seine, la 5 Quai de la 
Rapée, la 6 à Bercy. Sur 63 kilomètres une vingtaine sont inutilisables par excès d'eau et une quinzaine par défaut de courant. Démantelé, le réseau est dans l'incapacité de remplir sa mission.

\section{IX — LES MALHEURS DU CHEMIN DE FER}

Les cheminots n'ont pas lieu d'être fiers non plus. La première gare à fermer est la plus chic, la plus centrale : celle d'Orsay d'où les parlementaires du Sud-Ouest gagnent la Chambre des députés en 10 minutes. Le lundi 24 janvier la Seine envahit les voies en tranchée et les tunnels longeant les quais. Elle y pénètre en franchissant les parapets et en s'engouffrant dans les baies d'aération mal aveuglées. Cellesci ont été placées à 30 centimètres au-dessus du niveau maximum de la crue de 1876 que l'on croyait impossible à dépasser. Le fleuve coule donc dans une sorte de canal long de 3 kilomètres et demi entre le Jardin des Plantes et Orsay, le terminus. Par un regard les curieux aperçoivent une locomotive à vapeur naufragée, qui s'est laissé piéger.

Un kilomètre plus loin la Seine s'infiltre à nouveau sous les quais rive gauche et trouve un autre réservoir dans la gare des Invalides. De là, en suivant le ballast, elle file vers Grenelle et Issy-les-Moulineaux. Les deux têtes de ligne, celle d'Orléans et celle de l'Ouest resteront hors service durant 6 semaines.

Le 25 janvier la gare d'Austerlitz ferme à son tour car elle est menacée par des remontées du cours souterrain de la Bièvre. La ligne du Midi sera coupée également à Ivry et à Juvisy. Un peu plus tard c'est la gare de Lyon qui ferme car les voies sont submergées à Maisons-Alfort. Les compagnies transfèrent les départs d'Austerlitz à Denfert-Rochereau, ceux des Invalides à Saint-Lazare ou à Montparnasse et ceux de Lyon à la gare de l'Est et à la Bastille. Ces déplacements improvisés amènent la suppression de nombreux trains. Il devient fort compliqué de se rendre de Paris à Lyon, Marseille, Nice mais aussi à Montluçon, Bourges, Orléans, Toulouse, Angers, Nantes, Rennes et Brest. La capitale est pratiquement coupée des deux-tiers du territoire.

Même les têtes de ligne qui fonctionnent deviennent difficiles à atteindre. Devant Saint-Lazare, la Seine fait un surprenant barrage. Avant qu'elle ne ferme, la gare de Lyon était une presqu'île. Sur son parvis la Marine avait installé une sorte de port avec embarcadère et service de canots. Il fallait bien que les voyageurs partant ou arrivant franchissent les eaux.

\section{$X$ — LE MINISTÈRE DE L'INTÉRIEUR NE RÉPOND PLUS}

Le téléphone lui aussi montre sa fragilité. A partir du 24 janvier 1800 clients se plaignent de ne plus avoir de tonalité. Les communications régionales ne passent plus avec le SudOuest et le Sud-Est. On ne peut plus obtenir l'Espagne, le Portugal et l'Autriche. Cette situation empire jusqu'au 31 janvier. A cette date sur les 42000 lignes parisiennes, 14790 réparties dans une dizaine d'arrondissements sont hors-service ainsi que 140 circuits interurbains. L'eau s'est introduite dans les "chambres de coupures", locaux où les fils venant de chez les particuliers se connectent aux câbles de transport. Ceux-ci sont manchonnés de plomb et filent vers les centraux en passant par les égouts. Mais leur étanchéité est loin d'être parfaite. C'est au point que le ministère de l'Intérieur lui-même est coupé de la Préfecture de police.
Pour relier ces centres de commandement, les soldats des transmissions accrochent des lignes de campagne sur les arcades de la rue de Rivoli.

Ce qui est plus grave pour le commun des mortels, c'est que le télégraphe n'est plus fiable à l'intérieur comme à l'extérieur de la ville. Dix bureaux de poste sont atteints par les eaux et les pneumatiques qui transitaient par eux sont acheminés par des navettes automobiles. Pour l'extérieur la situation est plus sérieuse car les avaries viennent du poste central de la rue de Grenelle d'où rayonnent 728 câbles. L'eau ou simplement l'humidité y causent des ravages. Le 27 janvier 310 conducteurs sont en panne et 164 autres rendront l'âme dans les jours suivants. Paris ne communique plus avec les départements du Nord, du Nord-Ouest et du Sud-Est. Impossible non plus de télégraphier en Grande-Bretagne, en Belgique, aux Pays-Bas, au Danemark, en Suisse et en Italie. Ces dérangements durent un bon mois.

\section{XI — COMMENT S'EN DÉBARRASSER ?}

Les 1320 tonnes d'ordures quotidiennement ramassées dans la capitale sont emmenées normalement vers les usines de traitement situées à Saint-Ouen, Issy-les Moulineaux, Vitry et Romainville. Mais le lundi 24 janvier les tombereaux trouvent porte close à l'usine de Vitry, alors sous les eaux. Il va en être bientôt de même pour celles d'Issy et de Saint-Ouen. Impossible de diriger tous les charrois vers Romainville, site trop éloigné. Impossible également de recourir aux péniches et difficile de compter sur le chemin de fer. Mais alors comment débarrasser la capitale de ses déchets ? Le préfet de police Louis Lepine trouve la solution : les ordures ménagères seront déversées directement dans la Seine et emportées au loin par son flot exceptionnel et accéléré. La crue n'a pas que des inconvénients. Deux ponts sont affectés à ce qu'on pourrait appeler l'opération "ordures au fil de l'eau". En amont celui de Tolbiac, en aval le viaduc d'Auteuil (aujourd'hui disparu). Les déversements durent une quinzaine de jours. Des matières flottantes s'accrochent-elles dans les arches métalliques de certains ponts et les municipalités d'aval poussent-elles les hauts cris ? La troupe dégage les ponts et on fait la sourde oreille aux récriminations de la Seine inférieure.

\section{XII — UN HÔPITAL EVACUÉ EN CATASTROPHE}

La paralysie des services publics atteint également l'Assistance publique. L'établissement le plus menacé est l'hôpital Boucicaut situé dans le quartier de Javel, 15ème arrondissement, un secteur envahi dès le début de la crue. Il y a là 300 malades installés dans des pavillons en rez-de-chaussée. Le personnel construit des barrages mais l'inondation finit par s'infiltrer dans les sous-sols noyant le chauffage et les cuisines. Le 27 janvier à 4 heures du matin l'eau passe sous les portes des salles de malades. Le jour venu une noria de pompiers, d'agents de ville et d'infirmiers brancardent les alités vers d'autres hôpitaux parisiens.

On assiste aussi à l'envahissement progressif des soussols de cinq pavillons des Halles et les pompiers avec armes et bagages devront abandonner quatre de leurs casernes. Ne parlons pas du Jardin des Plantes dont les animaux sont condamnés à un bain permanent. 
Privés de moyens de transport et même de chaussée, gênés pour franchir la Seine puisqu'une demi douzaine de ponts sont interdits ou inaccessibles, nombre de Parisiens sont condamnés à rester chez eux. Beaucoup se font du souci pour leur réserves de vin, de pommes de terre et de charbon car environ 20000 caves sont inondées.

\section{XIII — L'ARCHEVÊQUE IMPLORE LE CIEL}

Le vendredi 28 janvier - un vendredi noir - la Seine est à son niveau maximum : 8 mètres 62 , soit 6 mètres au-dessus du niveau qui justifie une alerte et 2 mètres au-dessus de la crue qui faisait référence, celle de 1876. Paris est au bord de la paralysie totale. Sur les 41 usines indispensables au fonctionnement urbain (traitement des eaux, des ordures et des vidanges, fourniture d'air comprimé, de gaz et d'électricité) 23 sont noyées. Quatre gares sont closes, les tramways en déroute, le métro complètement désorganisé, 12500 lignes de teléphone en panne, le télégraphe à demi paralysé, l'air comprimé coupé, l'électricité et le gaz de plus en plus rares. Le gouvernement, l'administration, l'ensemble des services publics se sentent impuissants. L'angoisse atteint un tel degré que l'archevêque de Paris croit bon de dire un office à NotreDame pour implorer la clémence du ciel.

Miracle ? Le lendemain 29 janvier, après 12 jours de montée continue la Seine cesse de gonfler et même redescend de quelques centimètres. Mais les séquelles de la crue vont être longues à effacer. D'abord parce que le fleuve ne regagne définitivement son lit que le 15 mars 1910, deux mois après en être sorti. Ensuite parce que les réparations des réseaux les plus fragiles vont prendre beaucoup de temps. Le métro, les tramways et les trains repartent en peu de jours mais le télégraphe, le téléphone, l'électricité, l'air comprimé et le gaz d'éclairage tardent à revenir. Certains abonné ne recevront le courant que le 21 mai, 4 mois après les premières coupures.

Dans les rues atteintes par la crue, les pavés de bois sont partis à la dérive et les pavés de pierre se sont enfoncés sous l'effet des affouillements. La voirie a du pain sur la planche.

Les particuliers aussi car, après la crue, il faut faire la grande lessive de la capitale. Aux eaux terreuses de la Seine se sont mêlés les liquides encore plus douteux suintant des fosses de vidange. Une boue gluante et nauséabonde recouvre des centaines d'hectares. Gare aux épidémies. Les autorités prescrivent des mesures draconiennes et durant deux mois les Parisiens vident leurs caves, brossent les murs et désinfectent à l'aide de toutes sortes de produits distribués gratuitement. Certaines rues sont grattées et lavées de fond en comble six fois de suite. Finalement on estime que le nombre des immeubles touchés se situe entre 15 et 20000 et que 200000 Parisiens ont été directement lésés par l'inondation.

\section{XIII $\square$ FACTURE : HUIT MILLARDS DE FRANCS}

Curieusement on ne connait pas le montant des dégâts causés par la crue dans Paris intra-muros. On a davantage de données pour le département de la Seine qui englobait à l'époque les actuels Val-de-Marne, Hauts-de-Seine et SeineSaint-Denis. Une trentaine de communes ont été partiellement recouvertes par les eaux. Vingt et une ont été privées d'eau potable, 19 de gaz ou d'électricité, 14 ont imité la capitale en balançant leurs ordures à la Seine ; 190 usines y ont été condamnées au chômage mais surtout 15600 maisons ont été inondées et près de 400 pavillons se sont effondrés. Les dommages subis par le département sont évalués à 400 millions de francs-or auxquels il faut ajouter environ 50 millions de francs distribués à titre de secours. Le total équivaudrait à près de 8 milliards de francs 1997.

\section{BIBLIOGRAPHIE}

[1] 1910 PARIS INONDÉ, Marc Ambroise-Rendu, Editions Hervas, 1997, 111 p. 75 illust.

[2] RAPPORTS ET DOCUMENTS DIVERS, Commission des inondations, ministère de l'Intérieur, Imprimerie nationale, 1910, 709 p.

[3] Les inONDATIONS A PARIS EN 1910, Béatrice Camus, maîtrise d'histoire, Paris X-Nanterre, 1994, 189 p.

[4] COMPTES RENDUS dU CONSEIL. DE SURVEILl.ANCE DE L'ASSISTANCE PUBLIQUE, janvier, février, mars 1910. Archives de l'A.P.

[5] CRUES ET INONDATIONS, DOCUMENTS ADMINISTRATUFS, MANUSCRITS ET IMPRIMÉs. Archives de la Seine, 1910.

[6] PARIS AU JOUR LE JOUR. La presse quotidienne de 1910 à 1918", Elisabeth Hausser, 1968.

[7] Le Petrr Journal, janvier, février 1910. 